\title{
LENTIPES KAAEA, A NEW SPECIES OF FRESHWATER GOBY (TELEOSTEI: GOBIOIDEI: SICYDIINAE) FROM NEW CALEDONIA.
}

\author{
R.E. WATSON (1), P. KEITH (2), G. MARQUET (3)
}

(1) Goby Research, 3658 NW 41 $1^{\text {st }}$ Lane, GAINESVILLE, Florida 32605-1468, USA. E-mail : rewatson@bellsouth.net

(2) Laboratoire d'ichtyologie, Muséum National d'Histoire Naturelle, 43 rue Cuvier, F-75231 PARIS Cedex 05, France.

(3) L.E.R.V.E.M., Université Française du Pacifique, BP 4477, NOUMÉA, New Caledonia.

\begin{abstract}
Lentipes kaaea, new species, is described on the basis of 28 specimens collected from high gradient streams occurring in eastern slope region of North Province on New Caledonia and streams in the island nation of Vanuatu. Lentipes kaaea, new species, is distinguished from all other congeners by the following combination of characteristics: males and females with a single pair of fleshy lobes associated with and attached basally with urogenital papilla, lobes always apparent in males and only apparent in gravid females; usually 17 (17-18) pectoral rays; usually 10 (9-10) segmented rays in second dorsal fin; caudal peduncle with cycloid scales, few ctenoid scales may be present along midline in females, between belly and first dorsal fin ctenoid scales with 3 to 5 large spike like ctenii present on males and absent in females; cephalic sensory pore system with pores $\mathrm{A}, \mathrm{B}, \mathrm{C}, \mathrm{D}, \mathrm{F}, \mathrm{H}, \mathrm{K}, \mathrm{L}, \mathrm{N}$ and $\mathrm{O}$, pore $\mathrm{D}$ singular with all other pores paired, oculoscapular canal divided into posterior and anterior canals between pores $\mathrm{H}$ and $\mathrm{K}$; males bright red to purplish on head and on posterior half of body, second dorsal fin with a black spot between spine and ray 1 , individuals may have more than one spot present, first dorsal fin bluish to purplish and usually without spots, anal fin reddish to purplish basally and bluish distally; females usually whitish with variable tan to brownish markings that may include a distinct lateral band and a spot at medial base of caudal fin, above midline dusky bars and bands may be present, dusky saddles may be present dorsally, fin rays and spines with basal half slightly brownish and distally without pigment, membrane in all fins generally clear.
\end{abstract}

Key words : Lentipes kaaea, new species, New Caledonia, Vanuatu, Gobiidae, Sicydiinae. 


\title{
LENTIPES KAAEA, UNE ESPÈCE NOUVELLE DE GOBIE DULÇAQUICOLE DE NOUVELLE-CALÉDONIE (TELEOSTEI : GOBIOIDEI : SYCIDINAE).
}

\begin{abstract}
RÉSUMÉ
Lentipes kaaea, espèce nouvelle, est décrite à partir de 28 exemplaires collectés dans les rivières de la côte est de la Province nord de la Nouvelle-Calédonie et du Vanuatu. Cette espèce se distingue des autres par la combinaison des caractéristiques suivantes : mâles et femelles avec une seule paire de lobes charnus associés à la papille urogénitale, ces lobes étant toujours apparents chez le mâle et chez la femelle gravide ; 17 (17-18) rayons aux nageoires pectorales, 10 (9-10) rayons segmentés à la seconde nageoire dorsale ; le système de pores sensoriels céphaliques a les pores $A, B, C, D, F$, $\mathrm{H}, \mathrm{K}, \mathrm{L}, \mathrm{N}$ et $\mathrm{O}$, le pore D est simple alors que tous les autres sont doubles ; les mâles sont rouges brillants sur la tête et la partie médiane de la partie postérieure du corps, ils possèdent en général une tache noire sur la seconde dorsale tandis que la première n'en possède pas et est de couleur bleutée ; la nageoire anale est rosée avec sa marge distale bleutée à blanche ; la femelle est gris beige.
\end{abstract}

Mots-clés : Lentipes kaaea, espèce nouvelle, Nouvelle-Calédonie, Vanuatu, Gobiidae, Sicydiinae.

\section{INTRODUCTION}

For nearly a century Lentipes (Günther, 1861) was regarded as including two species, L. seminudus (Günther, 1880) and L. concolor (Gill, 1860), thought to be endemic to the Hawaiian Islands. Lentipes concolor, the type species for Lentipes, was originally placed into the genus Sicyogaster (Gill, 1860) but this name was found to be a junior homonym of a name preoccupied elsewhere in Pisces. MACIOLEK (1978) determined differences in recognizing the two species from the Hawaiian Islands as result of sexual dimorphism and placed $L$. seminudus into junior synonymy with $L$. concolor. Since the time of this important investigation (MACIOLEK, 1978) several species have been described; L. armatus (Sakai and Nakamura, 1979), L. bandama (Risch, 1980), L. rubrofasciatus (Maugé et al., 1992), L. whittenorum (Watson and Kottelat, 1994), L. watsoni (Allen, 1997), L. crittersius (Watson and Allen, 1999) and L. dimetrodon (Watson and Allen, 1999) and at least four more species have been discovered and await description.

Lentipes is currently known by 12 species, both described and undescribed, spanning a region from the Central and West Pacific and coastal streams of West Africa.

Lentipes has an unusual geographic distribution with $L$. bandama known from coastal drainages of West Africa and the remaining species occurring in insular streams of the Central and West Pacific. Lentipes bandama was originally placed into the monotypic genus Parasicydium (Risch, 1980) but there exist no characteristics that can clearly separate $L$. bandama from the remaining species at a genus level. PARENTI and MACIOLEK (1993) provisionally consider Parasicydium to be a junior synonym of Sicydium (Valenciennes, 1837) but this research strongly disagrees with that opinion based on numerous morphological and osteological differences that are not shared between the two genera. In Lentipes, to include Parasicydium, upper jaw teeth always sexually dimorphic in number of teeth and in possessing both tricuspid and conical to canine like teeth, (in Sicydium upper jaw teeth always of a single form and not sexually dimorphic in number), pectoral base strongly oblique at approximately $45^{\circ}$ with relationships to body axis (pectoral base slightly oblique at approximately $15^{\circ}$ with relationship to body axis), scales never 
present on nape and belly in adults (scales always present on nape and belly), pectoral rays 15-19, (pectoral rays 18-22), cephalic sensory pore system may be highly variable (cephalic sensory pore system stable with the only variation being the presence of two or three pores in the preopercular canal), cutaneous sensory papillae sexually dimorphic in distribution (cutaneous sensory papillae not sexually dimorphic in distribution), and ascending-articulating process on premaxilla sharply pointed at dorsal tip (ascendingarticulating process blunt to broadened at dorsal tip).

HARRISON (1993) considers two species from the Pacific Basin as belonging to Parasicydium but because of the broad overlap of characteristics that exist between all species now known to belong to Lentipes this research disagrees with that interpretation (HARRISON, 1993) as well.

Lentipes is unknown from any locality within the Indian Ocean basin suggesting the genus is far more ancient than contemporary research concerning the Gobioidei may suggest. In the western Indian Ocean Lentipes is replaced by Cotylopus (Guichenot, 1863) which share numerous morphological and osteological characteristics and is considered here its closest living relationship. It is suggested here that the absence of Lentipes from the Indian Ocean may be a result of geologic events that (1) closed the Tethys Sea, (2) impact the Indian Subcontinent has had since its collision with Asia and the subsequent uplift of the Himalayan Mountains resulting in dramatic climate changes in the northern Indian Ocean region, largely the presence of vast deserts, and (3) erosion of volcanic island chains to atolls resulting in a loss of suitable habitat for any Sicydiinae. Freshwater collection efforts on islands in the eastern Indian Ocean are scant and there exists a very real possibility that Lentipes may well exist in this region, especially islands off western shores of Sumatra, Java and the Malaysian Peninsula.

The primary purpose of this research is to describe Lentipes kaaea, new species, and to provide diagnoses concerning the genus Lentipes.

\section{METHODS}

Methods follow those in WATSON (1995). Measurements taken with a dial caliper measured to the nearest tenth of a millimeter. Morphometric data in tabular information rounded to the nearest whole percent of standard length (SL). Abbreviations used to represent institutions and collected cited follow LEVITON et al. (1985).

Comparative material used in establishing tabular information is not provided due to space constraints but a list is available by request from the author (REW).

\section{Lentipes Günther, 1861}

\section{Diagnosis}

First dorsal fin with six flexible spines, second dorsal fin with one flexible spine and usually nine or ten segmented rays, posterior most fin ray branched below skin at posterior most pterygiophore. Anal fins with one flexible spine and usually ten segmented rays, posterior most fin ray branched below skin at posterior most pterygiophore. Pelvic fins formed into strong a cup like disc adherent to belly between all five rays with one stout spine and five heavily branched rays, a strong fleshy frenum between both spines with a well developed circulatory supply clearly visible in living and fresh material. Pectoral fin with 15 to 19 rays depending on species; pectoral base strongly oblique positioned at approximately $45^{\circ}$ with relationship to body axis. Upper jaw always with tricuspid teeth anteriorly; posterior to tricuspid teeth recurved canine to canine like teeth in males and when present in females as small conical teeth; number of upper jaw teeth sexually 
dimorphic; all juveniles with only tricuspid teeth in upper jaw as males mature tricuspid teeth are shed and gradually replaced with canine to canine like teeth. Lower jaw with canine to canine like teeth in males and when present in females as small conical teeth, these teeth sexually dimorphic in number; a band of fine horizontal (labial) teeth present in lower jaw corresponding in position with tricuspid teeth of upper jaw. Cycloid scales always present at base of caudal fin; cycloid scales usually developed over caudal peduncle, when present ctenoid scales restricted to midline except for one species with several rows of midlateral ctenoid scales; males may have ctenoid scales between area of belly and first dorsal fin with large spike like ctenii, scales in this region usually absent in females and not present in males of all species. Cephalic sensory pore system variable and although present in all species certain pores and occasionally certain canals or portions of canals may be present or absent and may vary within an individual species. Cutaneous sensory papillae are sexually dimorphic in distribution with males having many more sensory papillae than females, especially along flanks where these may be well developed in males of some species and absent in females.

Lentipes kaaea, new species (Tables I-IV, Figures 1-4)

\section{Material examined}

28 specimens from New Caledonia and Vanuatu totalling 9 males and 19 females with a size range of $16.4-43.4 \mathrm{~mm} \mathrm{SL}$, largest male 29.9 , largest female 43.4 , smallest gravid female 30.7 .

\section{Holotype}

MNHN 1997-4175, male (26.1); New Caledonia: North Province: Napwé Imié above dam south of Napwéimiê near Poindimié; 13 Nov. 1997, G. Marquet and J.C. Guéry.

\section{Paratypes}

New Caledonia: North Province. MNHN 2002-113, 2 females (27.5-27.8), UF uncat., 1 male, 1 female (27.6-30.2); Napwé Imié; Oct. 1999, Chloé Expedition II. - MNHN 2002114, 3 males (21.7-26.5), MNHN 2002-115, 1 male, 9 females (25.5-43.4), UF uncat., 1 male, 1 female (20.5-26.3); rivière Kokengoné; 28 Oct. 1999, Chloé Expedition II. Vanuatu. MNHN 2002-147, 1 male, 5 females (16.4-27.4); Pentecost Island: Watmansabai River; 12 Oct. 1998, Vanuatu Department of Conservation. - SMF 28771, female (40.0); Epi Island: Peteview; 23 Jun. 1998, Vanuatu Department of Conservation. - SMF 29134, male (21.7); Pentecost Island: Bay Homo River; 9 Oct. 1998; Vanuatu Department of Conservation.

\section{Table I}

Number of pectoral rays in species of Lentipes.

\section{Tableau I}

Nombre de rayons aux nageoires pectorales chez les espèces de Lentipes.

\begin{tabular}{|c|c|}
\hline & 1516171819 \\
\hline L. armatus & 1128 \\
\hline L. bandama & 3143 \\
\hline L. concolor & 21277 \\
\hline L. crittersius & 1 \\
\hline L. dimetrodon & 5 \\
\hline L. kaaea & 244 \\
\hline L. rubrofasciatus & 1 \\
\hline L. whittenorum & $\begin{array}{lll}3 & 5 & 2\end{array}$ \\
\hline
\end{tabular}




\section{Table II}

Number of upper jaw teeth in species of Lentipes.

\section{Tableau II}

Nombre de dents à la mâchoire supérieure chez les espèces de Lentipes.

Tricuspid teeth

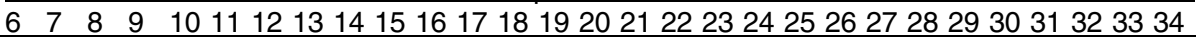

L. armatus males

L. armatus females

L. bandama males

L. bandama females

L. concolor males

L. concolor females

L. crittersius female

L. dimetrodon males

L. dimetrodon female

L. kaaea males

L. kaaea females

L. rubrofasciatus males

L. whittenorum males

L. whittenorum females

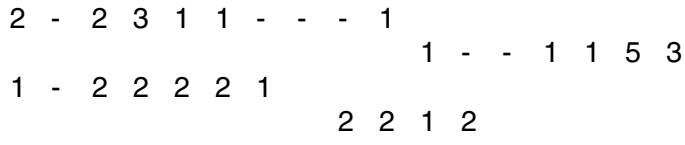

$2 \begin{array}{llll}- & 1 & 1 & 2\end{array}$

\section{Table III}

Zigzag scale counts in species of Lentipes.

\section{Tableau III}

Nombre d'écailles en zigzag chez les espèces de Lentipes.

\begin{tabular}{|c|c|c|c|c|c|c|c|c|c|c|c|c|c|c|c|c|}
\hline & 0 & 1 & 2 & 3 & 4 & 5 & 6 & 7 & 8 & 9 & 1011 & 12 & 13 & 14 & & 1617 \\
\hline L. armatus & & & & & & & & & & 5 & 94 & 2 & 1 & & & \\
\hline L. bandama & & & & & & & & & & & 2 & 2 & 7 & 6 & 3 & \\
\hline L. concolor & 2 & - & 1 & - & 5 & 3 & 6 & 5 & 7 & 6 & 5 & 2 & 4 & 1 & 2 & 1 \\
\hline L. crittersius & & & & & & & & & & & 1 & & & & & \\
\hline L. dimetrodon & & & & & & & & & 1 & 3 & 1 & & & & & \\
\hline L. kaaea & 2 & - & - & - & - & - & - & - & - & 7 & 6 & 2 & & & & \\
\hline L. rubrofasciatus & & & & & & & & 2 & - & 4 & 1 & & & & & \\
\hline L. whittenorum & & & & & & & & & & 3 & 3 & 1 & 1 & 1 & 1 & \\
\hline
\end{tabular}




\section{Table IV}

Morphometrics in species of Lentipes expressed to the nearest whole percent of standard length.

\section{Tableau IV}

Morphométrie chez les espèces de Lentipes exprimée en pourcentage (entier) de la longueur standard.

\begin{tabular}{|c|c|c|c|c|c|c|c|c|c|c|c|c|c|c|c|}
\hline & \multicolumn{15}{|c|}{ Predorsal length } \\
\hline & 32 & 33 & 34 & 35 & 36 & 37 & 38 & 39 & 40 & 41 & 42 & 43 & 44 & 454 & 46 \\
\hline L. armatus & 1 & - & - & - & - & 2 & 3 & 7 & 6 & 2 & & & & & \\
\hline L. bandama & & 1 & 1 & 5 & 9 & 2 & 2 & & & & & & & & \\
\hline L. concolor & & 1 & 4 & 9 & 17 & 7 & 6 & 1 & & & & & & & \\
\hline L. crittersius & & & & & & & 1 & & & & & & & & \\
\hline L. dimetrodon & & & & & 1 & - & 4 & 1 & & & & & & & \\
\hline L. kaaea & & & & & 1 & 4 & 4 & 7 & 4 & 3 & 1 & 1 & 1 & - & 1 \\
\hline L. rubrofasciatus & & 2 & - & - & 2 & 2 & - & 1 & - & 1 & & & & & \\
\hline L. whittenorum & & & & & & 6 & 4 & & & & & & & & \\
\hline
\end{tabular}

\begin{tabular}{llllllllllllll}
\hline & \multicolumn{11}{c}{ Preanal length } \\
\cline { 2 - 3 } & 53 & 54 & 55 & 56 & 57 & 58 & 59 & 60 & 61 & 62 & 63 & 64 & 65 \\
\hline L. armatus males & & & & & 1 & - & 4 & 3 & 1 & 2 & & & \\
L. armatus females & & & & & & & 1 & - & 4 & 2 & 3 & 1 & \\
L. bandama males & & & 1 & 4 & 4 & 1 & 1 & & & & & \\
L. bandama females & & & 2 & 1 & - & 3 & - & 1 & 1 & 1 & & & \\
L. concolor males & & & & 1 & - & 2 & 1 & 9 & 4 & 2 & & & \\
L. concolor females & & & 1 & 1 & 1 & 2 & 5 & 6 & 5 & 7 & 2 & - & 1 \\
L. crittersius female & & & & & & & & & & 1 & & & \\
L. dimetrodon males & 1 & - & - & - & - & - & 3 & 1 & & & & & \\
L. dimetrodon female & & & & & & & & 1 & & & & & \\
L. kaaea males & & & & & & & 1 & - & 3 & 2 & 1 & 1 & 1 \\
L. kaaea females & & & 1 & - & 2 & - & 3 & 2 & 5 & 4 & 1 & & \\
L. rubrofasciatus males & & 1 & - & 2 & - & 2 & 1 & 1 & & & & \\
L. whittenorum males & & & & & & & & 1 & - & - & 2 & \\
L. whittenorum females & & & & & & & 1 & 3 & 2 & 1 & &
\end{tabular}

\begin{tabular}{|c|c|c|c|c|c|c|c|c|}
\hline & \multicolumn{8}{|c|}{ Head length } \\
\hline & 1920 & 21 & 22 & 23 & 24 & 25 & 26 & 27 \\
\hline L. armatus males & & & & & 3 & 6 & 1 & \\
\hline L. armatus females & & & 8 & 3 & & & & \\
\hline L. bandama males & & & 4 & 6 & 2 & & & \\
\hline L. bandama females & & & 1 & 6 & 1 & 1 & & \\
\hline L. concolor males & & & 1 & 4 & 7 & 4 & 3 & \\
\hline L. concolor females & & 3 & 10 & 11 & 6 & - & 1 & \\
\hline L. crittersius female & & & 1 & & & & & \\
\hline L. dimetrodon males & & & & & 2 & 2 & 1 & \\
\hline L. dimetrodon female & & & & & 1 & & & \\
\hline L. kaaea males & & & & & 2 & 5 & 1 & 1 \\
\hline L. kaaea females & 3 & 1 & 7 & 5 & 1 & 1 & & \\
\hline L. rubrofasciatus males & & & & 1 & 5 & 1 & 1 & \\
\hline L. whittenorum males & & & & 1 & 1 & - & 1 & \\
\hline L. whittenorum females & & & 2 & 5 & & & & \\
\hline
\end{tabular}




\begin{tabular}{|c|c|c|c|c|c|c|c|c|}
\hline & & & & Jav & $v$ len & gth & & \\
\hline & 7 & 8 & 9 & 10 & 11 & 12 & 13 & 14 \\
\hline L. armatus males & & & & & & & 9 & 1 \\
\hline L. armatus females & & & 3 & 7 & 1 & & & \\
\hline L. bandama males & & & & 5 & 5 & & & \\
\hline L. bandama females & & 2 & 5 & 1 & 2 & & & \\
\hline L. concolor males & & & & 2 & 5 & 12 & & \\
\hline L. concolor females & 2 & 7 & 12 & 9 & 1 & & & \\
\hline L. crittersius female & & 1 & & & & & & \\
\hline L. dimetrodon males & & & & 1 & 2 & 2 & & \\
\hline L. dimetrodon females & & 1 & & & & & & \\
\hline L. kaaea males & & & & & 5 & 2 & 2 & \\
\hline L. kaaea females & 3 & 4 & 6 & 5 & & & & \\
\hline L. rubrofasciatus males & & & & 4 & 1 & 2 & 1 & \\
\hline L. whittenorum males & & & & & & 1 & 1 & 1 \\
\hline L. whittenorum females & & 6 & - & 1 & & & & \\
\hline
\end{tabular}

Caudal peduncle length

L. armatus males

L. armatus females

L. bandama males

L. bandama females

L. concolor males

L. concolor females

L. crittersium female

L. dimetrodon males

L. dimetrodon female

L. kaaea males

L. kaaea females

L. rubrofasciatus males

L. whittenorum males

L. whittenorum females

$\begin{array}{llllllll}15 & 16 & 17 & 18 & 19 & 20 & 21 & 22\end{array}$

\begin{tabular}{|c|c|c|c|c|c|}
\hline & & unda & $p \in$ & dunc & le depth \\
\hline & 7 & 8 & 9 & 10 & 1112 \\
\hline L. armatus males & & & 1 & 3 & 6 \\
\hline L. armatus females & & & 3 & 6 & 1 \\
\hline L. bandama males & & & 7 & 4 & \\
\hline L. bandama females & & 4 & 5 & & \\
\hline L. concolor males & & 1 & 6 & 6 & 5 \\
\hline L. concolor females & 5 & 18 & 9 & & \\
\hline L. crittersius female & & & 1 & & \\
\hline L. dimetrodon males & & & & 1 & 2 \\
\hline L. dimetrodon female & & & & & 1 \\
\hline L. kaaea males & & & 3 & 5 & 1 \\
\hline L. kaaea females & & & 9 & 9 & \\
\hline L. rubrofasciatus males & & & 3 & 3 & 1 \\
\hline L. whittenorum males & & & & 2 & 1 \\
\hline L. whittenorum females & & & & 6 & 1 \\
\hline
\end{tabular}

$\begin{array}{lllllll} & & & 6 & 4 & & \\ 1 & 2 & 4 & 6 & 1 & & \\ 1 & 3 & 3 & 2 & 1 & & \\ 1 & 6 & 7 & 2 & 2 & - & 1 \\ 1 & 9 & 3 & 14 & 3 & 2 & \\ & & & 1 & & & \\ & & 1 & 2 & 1 & 1 & \\ & & & 1 & & & \\ 2 & 2 & 2 & 2 & 1 & & \\ & 1 & 1 & 4 & 7 & 3 & 2 \\ 3 & - & 1 & 1 & & & \\ 2 & 1 & & & & & \\ 1 & - & 3 & 2 & 1 & & \end{array}$

Body depth in males at second dorsal origin

$\begin{array}{llllllllll}11 & 12 & 13 & 14 & 15 & 16 & 17 & 18 & 19 & 20\end{array}$

L. armatus

$\begin{array}{llllllll}1 & - & 1 & 1 & 1 & 2 & 2 & 2\end{array}$

L. bandama

$\begin{array}{llll}2 & 2 & 5 & 2\end{array}$

L. concolor

$\begin{array}{lllll}2 & 8 & 4 & 3 & 1\end{array}$

L. dimetrodon

L. kaaea

L. rubrofasciatus

$2 \quad 2 \quad 3 \quad-1-1 \quad-1$

L. whittenorum

$\begin{array}{lllllll}3 & 1 & 1 & & & \\ & & 1 & - & - & \end{array}$


Second dorsal fin length

\begin{tabular}{|c|c|c|c|c|c|c|c|c|c|c|c|c|c|c|c|c|}
\hline & 25 & 26 & 27 & 28 & 29 & 30 & 31 & 32 & 33 & 34 & 35 & 36 & 37 & 38 & 39 & 40 \\
\hline L. armatus males & & & & & 1 & 1 & 2 & 3 & 1 & 2 & & & & & & \\
\hline L. armatus females & & 1 & 4 & 4 & 2 & & & & & & & & & & & \\
\hline L. bandama males & & & & & & & & 1 & 2 & 6 & 1 & 1 & & & & \\
\hline L. bandama females & & & & & 1 & 5 & 1 & - & 2 & & & & & & & \\
\hline L. concolor males & & & & & & 2 & - & - & 1 & 4 & 4 & 3 & 2 & 2 & 1 & \\
\hline L. concolor females & 1 & 1 & 1 & 5 & 5 & 10 & 6 & 1 & & & & & & & & \\
\hline L. crittersius female & & & & & & 1 & & & & & & & & & & \\
\hline L. dimetrodon males & & & & & & & & & & 1 & - & 1 & - & 1 & - & 2 \\
\hline L. dimetrodon female & & & 1 & & & & & & & & & & & & & \\
\hline L. kaaea males & & & & & & 4 & 2 & 3 & 1 & & & & & & & \\
\hline L. kaaea females & 3 & 1 & 5 & 5 & 1 & - & 1 & & & & & & & & & \\
\hline L. rubrofasciatus males & & & & & & & 1 & - & - & 2 & - & 2 & 1 & 1 & - & 1 \\
\hline L. whittenorum males & & & & & & & & 1 & - & 1 & - & 1 & & & & \\
\hline L. whittenorum females & & & & 2 & 2 & 2 & - & 1 & & & & & & & & \\
\hline
\end{tabular}

Anal fin length

\begin{tabular}{|c|c|c|c|c|c|c|c|c|c|c|c|c|c|c|c|c|c|c|c|}
\hline & \multicolumn{19}{|c|}{ Anal fin length } \\
\hline & 21 & 22 & 23 & 24 & 25 & 26 & 27 & 28 & 29 & 30 & 31 & 32 & 33 & 34 & 35 & 36 & 37 & 38 & 39 \\
\hline L. armatus males & & & & & & & 1 & 2 & 3 & 3 & - & 1 & & & & & & & \\
\hline L. armatus females & & & 1 & - & 5 & 3 & 2 & & & & & & & & & & & & \\
\hline L. bandama males & & & & & & & & & & & 4 & 6 & 1 & & & & & & \\
\hline L. bandama females & & & & & & 2 & 3 & 3 & 1 & & & & & & & & & & \\
\hline L. concolor males & & & & & & & & 1 & 4 & 6 & 3 & 3 & 2 & & & & & & \\
\hline L. concolor females & 1 & - & 1 & 6 & 6 & 10 & 4 & 1 & 1 & & & & & & & & & & \\
\hline L. crittersius female & & & & & & 1 & & & & & & & & & & & & & \\
\hline L. dimetrodon males & & & & & & & & & & & & & & 1 & 1 & 1 & 1 & - & 1 \\
\hline L. dimetrodon female & & & & & & & & 1 & & & & & & & & & & & \\
\hline L. kaaea males & & & & & 2 & 4 & 1 & 1 & 1 & 1 & & & & & & & & & \\
\hline L. kaaea females & & & 2 & 4 & 4 & 5 & 2 & & & & & & & & & & & & \\
\hline L. rubrofasciatus males & & & & & & & & & & 2 & 1 & 2 & 1 & - & 1 & - & 1 & & \\
\hline L. whittenorum males & & & & & & & 1 & 2 & & & & & & & & & & & \\
\hline L. whittenorum females & 2 & 1 & 1 & 2 & - & 1 & & & & & & & & & & & & & \\
\hline
\end{tabular}

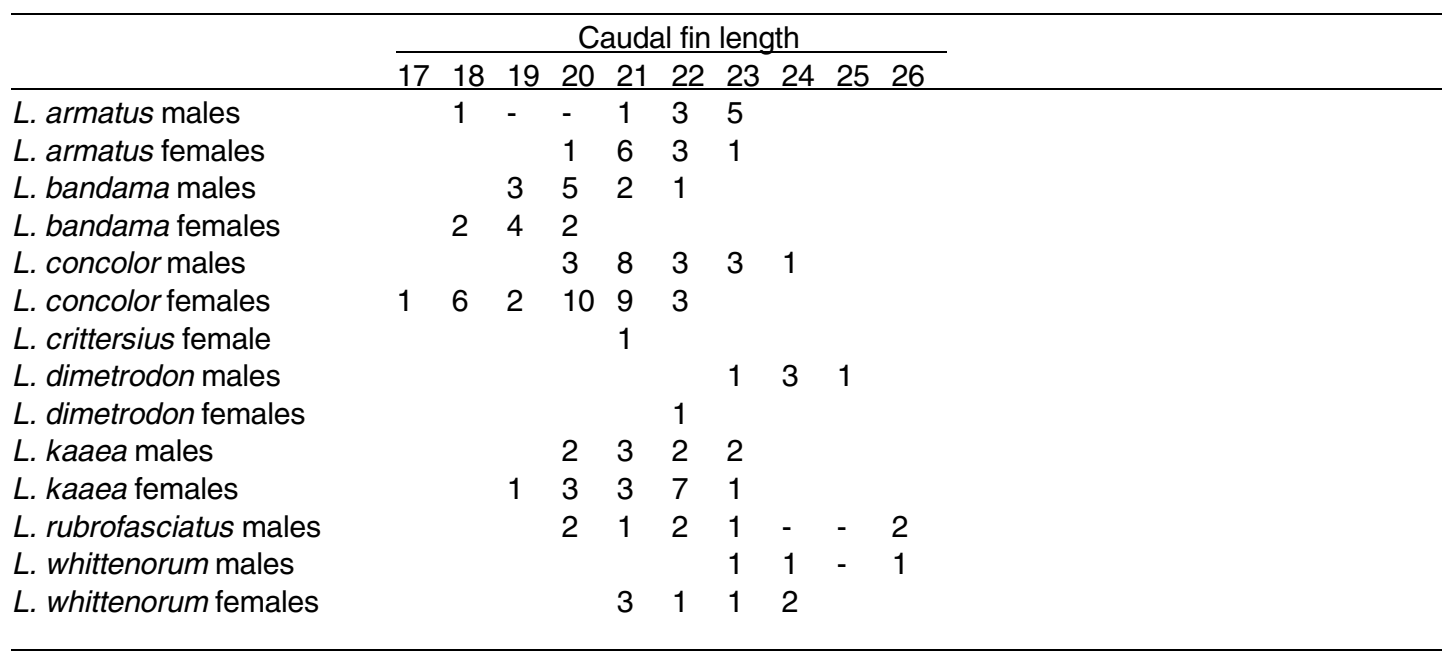

\section{Diagnosis}

Second dorsal fin rays usually 10 (9-10) rays. Pectoral rays usually 17 (17-18). Slightly flexible tricuspid teeth in upper jaw, males with recurved canine like teeth in upper and lower jaws, females usually without conical teeth in upper or lower jaws. Males with snout, upper lip, second dorsal fin, and area between second dorsal and anal fins bright 
red to purplish; second dorsal fin with a black spot adjacent to ray 1 medially, red usually not touching spot; anal fin usually reddish basally bluish distally; a pair of lobes associated with urogenital papilla, paired lobes adjacent to urogenital apparent only in gravid material. Females variable ranging from mostly whitish appearing slightly dusky with or without a blackish spot medially at base of caudal fin to having an irregular midlateral band with various dusky streaks and/or bars above midline with square shaped dusky saddles.

\section{Description}

Dorsal fins almost always VI-I, 10 (9-10), membrane on first dorsal fin not touching base of second dorsal fin, spines not filamentous. Anal fins almost always I, 10 (9-10) and directly opposite to second dorsal fin. Pectoral fins almost always with 17 (17-18) rays, ventral most 1 or 2 rays simple, posterior margin rounded. Cycloid scales thin and slightly embedded on caudal peduncle, few cycloid scales extend anteriorly along midline between second dorsal and anal fins, in both sexes scales usually present along base of second dorsal fin either partially or entirely and in females usually along base of anal fin, posterior to hypural base with few cycloid scales, in some females few ctenoid scales may be present along midline; in males ctenoid scales with large spike like ctenii mostly obscured by pectoral fin on trunk present ventral to first dorsal fin and dorsal to belly, scales of near equal size with large ctenii numbering 3 to 5 along posterior margin, between ctenoid and cycloid scales a fairly large naked region; zigzag scales 9-12. Head, breast, nape and belly without scales. Upper jaw teeth distinctly tricuspid anteriorly and slightly flexible; males usually with 4 (4-5) recurved canine like teeth posterior to tricuspid teeth; females usually without teeth posterior to tricuspid teeth, when present not more than one and always much smaller than in males. Teeth in lower jaw recurved and canine like teeth in males (4-5), females usually without lower jaw teeth when present with up to 4 small conical teeth. Rakers on inner edge of outer gill arch appear as a small papillous projection without ossification (0+0+1). Cephalic sensory pore system A, B, C, D, F, H, K, L, N and O, pore D singular with all others paired (Figure 1), oculoscapular canal divided into anterior and posterior canal between pores $\mathrm{H}$ and $\mathrm{K}$. Cutaneous sensory papillae not well developed on head, sensory papillae present between pores $\mathrm{H}$ and $\mathrm{K}$; males with vertical rows of papillae along flanks and sometimes on caudal peduncle, sensory not present on trunk or caudal peduncle in females. Urogenital papilla in males slender and pointed distally (Figure 2a), a pair of fairly broad and flattened lobes obscure most of urogenital papilla in breeding males, urogenital papilla not retractable into a sheath like groove; in females urogenital papilla rectangular in appearance (Figure 2b) not retractable into a sheath like groove, 2 curved lobes not apparent except in gravid material.

\section{Color in preservation}

Males: Body grayish, between second dorsal and anal fins slightly dusky. First dorsal fin appears grayish to blackish, a spot or spots may form a weak midlateral band. Second dorsal fin with a black spot medially at ray 1, membrane between spine and ray 1 mostly without pigment, two or more spots occasionally present. Caudal fin with rays dusky, membrane mostly without pigment. Anal fin basal half clear, medially a black band from spine to ray 10. Pelvic disc dusky. Pectoral fin and pectoral base slightly dusky.

Females: Mostly white. Head and body dusky laterally being darkest midlaterally on caudal peduncle, an irregular lateral band sometimes present, above midline some dusky bars and bands may be present, dorsally few dusky square shaped saddles may be present. Opercle with a blackish triangular patch. Blackish spot medially at base of caudal fin may be absent in some specimens. Dorsal rays and spines dusky, membrane and rays distally without pigment. Caudal fin rays dusky, membrane and distal margin without pigment. Anal fin with blackish pigmentation at base of rays and spine, remainder not pigmented. Pelvic disc not pigmented. Pectoral rays and membrane dusky. Pectoral base dusky with a medial blackish blotch. 

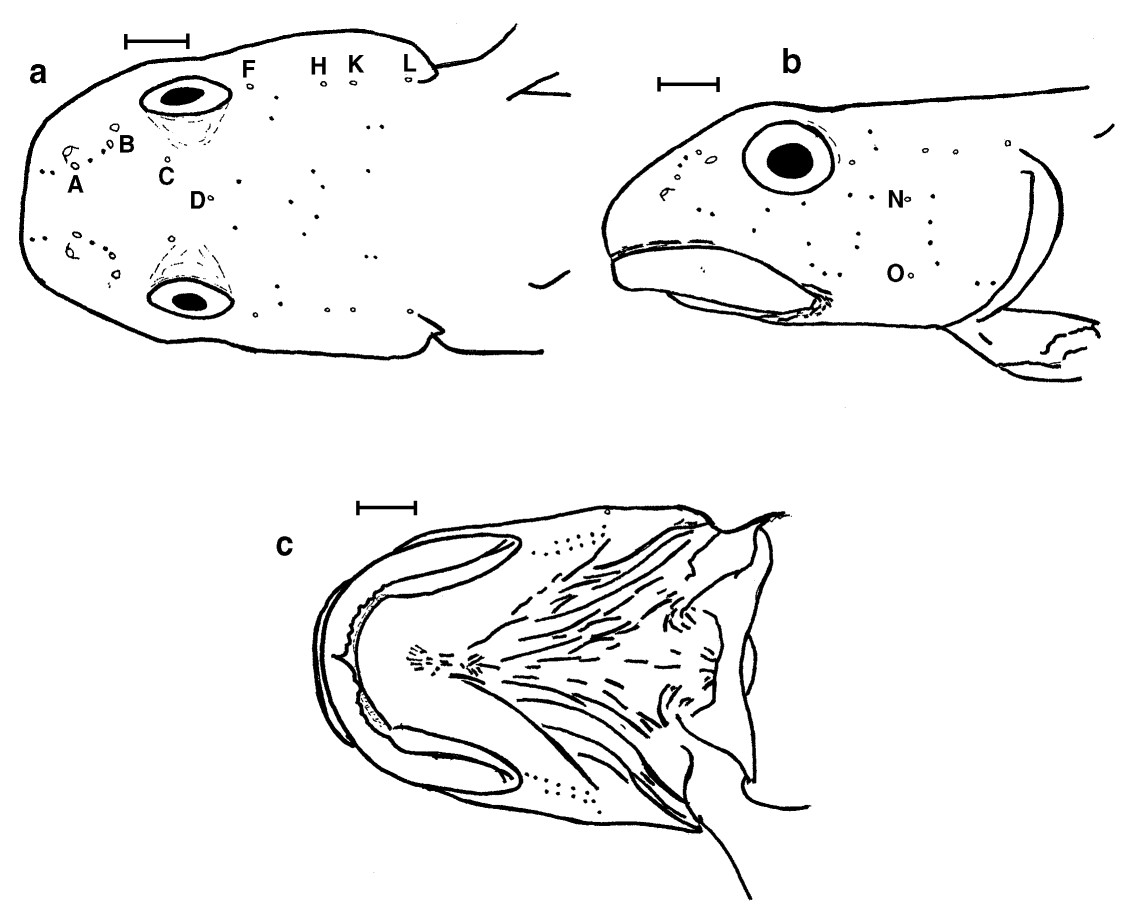

Figure 1

Diagrammatic illustration of head in Lentipes kaaea showing head pores and sensory papillae: a. dorsal view; b. lateral view; c. ventral view.

\section{Figure 1}

Illustration schématique de la tête de Lentipes kaaea montrant les pores céphaliques et les papilles sensorielles : $a$. vue dorsale ; b. vue latérale ; $c$. vue ventrale.
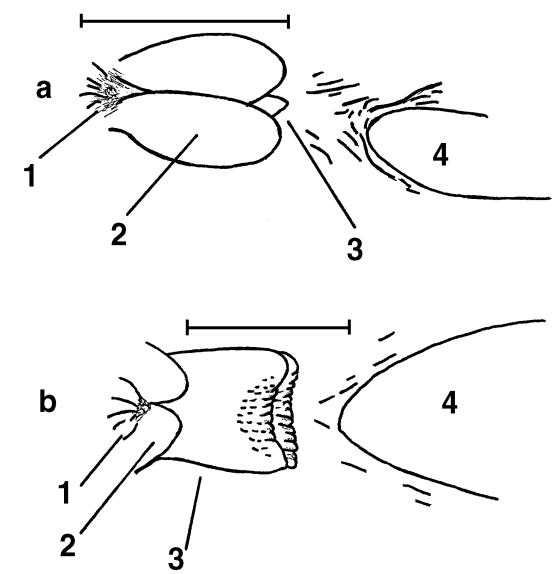

Figure 2

Diagrammatic illustration of urogenital papilla in Lentipes kaaea : a. male;

b. female; 1. anus; 2. urogenital lobes; 3. urogenital papilla; 4. anal fin.

\section{Figure 2}

Illustration schématique des papilles urogénitales de Lentipes kaaea : a. mâle ; b. femelle ; 1. anus ; 2 . lobe urogénital ; 3. papille urogénitale ; 4. nageoire anale. 


\section{Color in life}

Males (Figure 3a): Second dorsal fin and area between second dorsal bright red to purplish; upper lip and snout red to purplish; trunk with blackish blue bars present on ctenoid scales; basal half of anal fin reddish to purplish remainder bluish.

Females (Figure 3b): Tan to brownish with dusky markings appearing similar to that in preservation.

\section{Distribution}

Lentipes kaaea is known from eastern slope streams of North Province, New Caledonia and the island nation of Vanuatu.

\section{Ecology}

Lentipes kaaea occurs in swift clear high gradient streams with slab and boulder strewn bottom, some course gravel may be present. Lentipes kaaea has been observed swimming in strong currents rather than being attached to rock surfaces like other gobies belonging to Sicydiinae found in the same environment.

\section{Affinities}

Based on lobes adjacent to urogenital papilla it shares some affinities with L. rubrofasciatus (Maugé et al., 1992) and L. whittenorum but lobes not as broadly developed. Black spot on first dorsal fin and large ctenii on anterior scales resembles L. armatus (Sakai and Nakamura, 1979), L. watsoni (Allen, 1997) and an undescribed species from Bali, Indonesia, but $L$. kaaea differs immediately in having urogenital lobes.

\section{Etymology}

The new species is named for the Napwé Tribal Chief Kaaea who kindly permitted collection of freshwater fishes on tribal lands. Kaaea is a name used in Melanesia and throughout Polynesia, to include Hawaii the type locality for Lentipes, and is used here to exemplify the broad distribution of Lentipes. The new species name is treated as a noun in apposition.

\section{ACKNOWLEDGEMENTS}

We thank the following for help during Chloé 1, 2 and 3 Expeditions: E. Vigneux (High Fisheries Council), E. Feunteun (University of Rennes), N. Mary (University of Nouméa), P. Sasal (University of Perpignan), J. Manauté (Environmental Department of Province Nord), F. Devinck (Environmental Department of Province Sud) and the Vanuatu Department of Conservation, especially L. Nimoho, for their interest and concerns for the conservation of flora and fauna native to Vanuatu.

\section{REFERENCES}

ALLEN G.R., 1997. Lentipes watsoni, a new species of freshwater goby (Gobiidae) from Papua New Guinea. Ichthyological Exploration of Freshwaters, 8, 33-40.

GILL T.N., 1860. Conspectus piscium in expeditione ad oceanum Pacificum septendrionelum, C. Ringoldio et J. Rodgersio dusibus, a Guielmo Stimpsono collectorum. Sicydianae. Proceedings of the Academy of Natural Sciences of Philadelphia, 12, 100-102. 
GUICHENOT A., 1863. Faune ichthyologique. In : Maillard L., Notes sur l'île de la Réunion. Paris, $32 \mathrm{p}$.

GÜNTHER A., 1861. Catalogue of the Acanthopterygian fishes in the collection of the British Museum. Vol. III. British Museum, London, 586 p.

GÜNTHER A., 1880. Report on the shore fishes procured during the voyage of H. M. S. Challenger in the years 1873-1876. 81 p. In : The voyage of the H. M. S. Challenger. Zoology, British Museum, London.

HARRISON I.J., 1993. The West African sicydiine fishes, with notes on the genus Lentipes (Teleostei: Gobiidae). Ichthyological Exploration of Freshwaters, 4, 201-232.

LEVITON A.E., GIBBS R.H., HEAL E., DAWSON C.E., 1985. Standards in herpetology and ichthyology: Part I. Standard symbolic codes for institutional resource collections in herpetology and ichthyology. Copeia, 1985, 802-832.

MACIOLEK J.A., 1978. Taxonomic status, biology, and distribution of Hawaiian Lentipes, a diadromous goby. Pacific Science, 31, 355-362.

MAUGÉ A., MARQUET G., LABOUTE P., 1992. Les Sicydiinae (Gobiidae) des eaux douces de la Polynésie française. Description de trois espèces nouvelles. Cybium, 16, 213-231.

PARENTI L.R., MACIOLEK J.A., 1993. New sicydiine gobies from Ponape and Palau, Micronesia, with comments of systematics of the subfamily Sicydiinae (Teleostei: Gobiidae). Bulletin of Marine Science, 53, 945-972.

RISCH L., 1980. Description of Parasicydium bandama, gen. nov., sp. nov., a new gobiid fish from the Bandama River, Ivory Coast (Pisces Gobiidae). Revue zoologie d'Afrique, 94, 126-132.

SAKAI H., NAKAMURA M., 1979. Two new species of freshwater gobies (Gobiidae: Sicydiaphinae) from Ishigaki Island, Japanese Journal of Ichthyology, 26, 43-54.

VALENCIENNES A., 1837. Tome douzième. In : CUVIER G. and VALENCIENNES A., Histoire naturelle de poissons. Levrault, Strasbourg, $507 \mathrm{p}$.

WATSON R.E., 1995. Gobies of the genus Stiphodon from French Polynesia, with descriptions of two new species (Teleostei: Gobiidae: Sicydiinae). Ichthyological Exploration of Freshwaters, 6, 33-48.

WATSON R.E., ALLEN G.R., 1999. New species of freshwater gobies from Irian Jaya, Indonesia (Teleostei: Gobioidei: Sicydiinae). Aqua - Journal of Ichthyology and Aquatic Biology, 3, 113-118.

WATSON R.E., KOTTELAT M., 1994. Lentipes whittenorum and Sicyopus auxilimentus, two new species of freshwater gobies from the western Pacific (Teleostei: Gobiidae: Sicydiinae). Ichthyological Exploration of Freshwaters, 5, 351-364. 
a

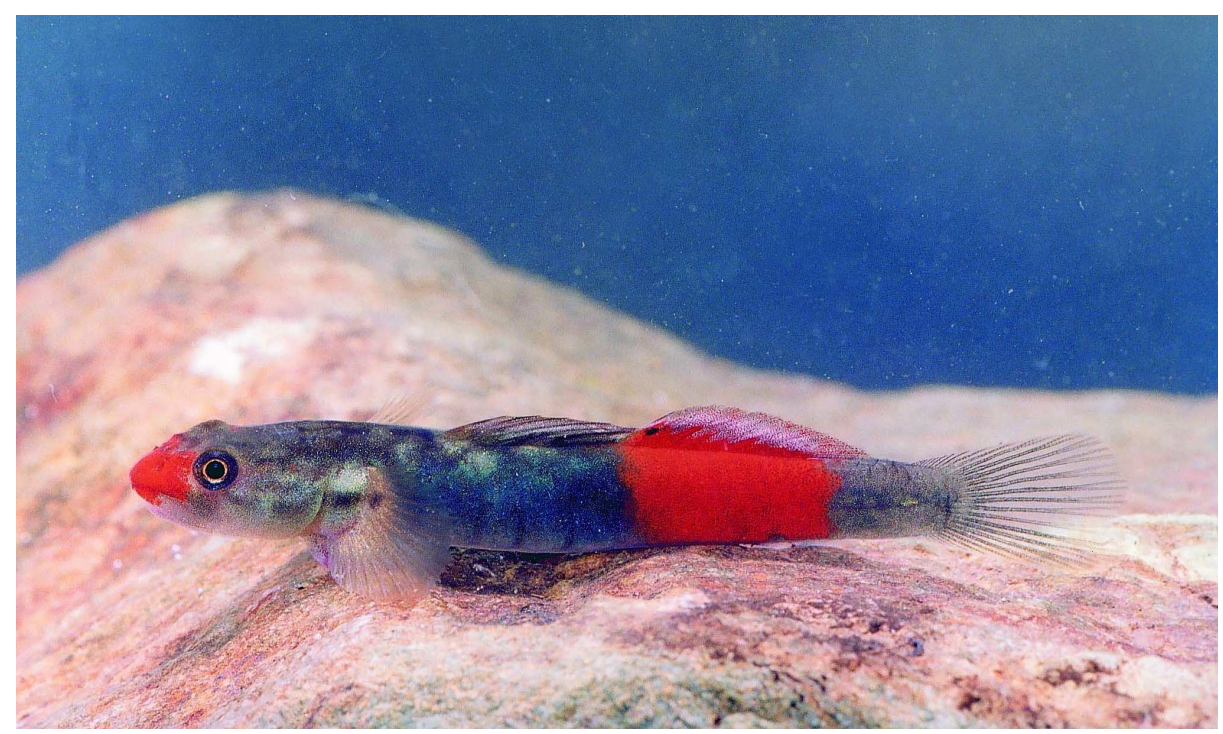

b

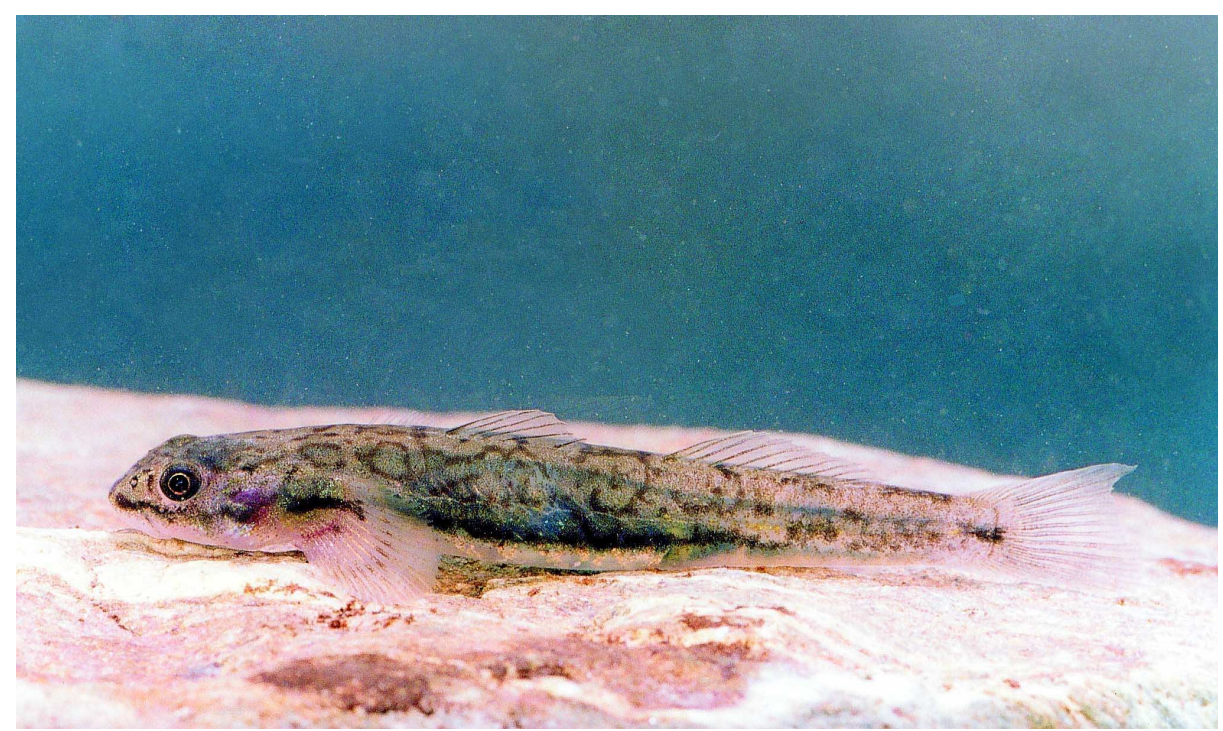

Figure 3

Lentipes kaaea, New Caledonia: a. male, Napwéimié River (picture by E. Vigneux MNHN/CSP - Chloé I); b. female, Napwéimié River (picture by E. Vigneux MNHN/CSP - Chloé I).

Figure 3

Lentipes kaaea, Nouvelle-Calédonie: a. mâle, rivière Napwéimié (photo de

E. Vigneux MNHN/CSP - Chloé I) ; b. femelle, rivière Napwéimié (photo de E. Vigneux MNHN/CSP - Chloé I). 
